CREAT. MATH. INFORM.

Volume 25 (2016), No. 2,

Pages 159 - 164
Online version at https : //creative-mathematics. cunbm. utcluj.ro/

Print Edition: ISSN 1584 - 286X; Online Edition: ISSN 1843 - 441X

DOI: https://doi.org/10.37193/CMI.2016.02.06

\title{
Relationship between open sets with respect to an ideal
}

\author{
CARlos Carpintero $^{1}$, Jackeline PacheCo ${ }^{2}$ and EnNis Rosas ${ }^{1,2}$
}

\begin{abstract}
In this article, we obtain the relationship between the open sets with respect to an ideal defined by [ Michael, F. I., On some open sets with respect to an ideal, Eur. J. Pure Appl. Math, 6 (2013), No. 1, 53-58.], [Rodyna, A. Hosny and Deena, Al-Kadi, Types of Generalized Open Sets with Ideal, Int J Comput Appl., 80 (2013), No. 4, 97-112.], [Rodyna, A. Hosny, Pre-open sets respect ideal, Eur. J. Sci. Res., 104 (2013), No. 1, 99-101.], and the open sets with respect to an ideal defined by [Rosas, E., Carpintero, C., Muñoz, A., Pacheco, J., Some Remarks on Semi Open Sets with Respect to an Ideal, Eur. J. Pure Appl. Math, 7 (2014), No. 4, 437-441.] and [Arafa A. N., Mareay, R. Michael, F. I., Idealization of Some Topological Concepts, Eur. J. Pure Appl. Math, 8 (2015), No. 3, 389-394.]. Also we introduce another class of open sets with respect to an ideal called $I$ - $b$-open set and we give the relationships between the three last one.
\end{abstract}

\section{INTRODUCTION AND PRELIMINARIES}

In 1963, the notion of semi open sets was introduced by Levine [5]. In 2013, Friday Ifeanyi Michael [8], studied the semi open sets with respect to an ideal. In 2013, Rodyna A. Hosny et al. [11], introduced and studied the notions of $\alpha$-open sets and $\beta$-open sets with respect to an ideal $I$. Again in 2014, Rodyna A. Hosny et al. [10], introduced and studied the pre-open sets with respect to an ideal $I$, and give the existent relations between the sets described above. In 2014, C. Carpintero et al. [12], introduced and characterized the notions of weakly semi open sets with respect to an ideal $I$ and finally, in 2015, Arafa A. Nasef et al. [2], defined and studied the $I-\beta$ - open sets. In this article, we obtain the existent relation between the sets defined in [8],[11],[10] with the sets defined in [12] and [2], also, we define in a natural form the $I$ - $b$-open set as a generalization of the $b$-open sets. Finally we looking for the relation between the sets defined in [12], [2] and the $I$-b-open set.

\section{SETS WITH RESPECT TO AND IDEAL}

Throughout this paper $(X, \tau)$ (or simply $X$ ) represent a topological space on which no separation axioms are assumed unless otherwise mentioned. For a subset $A \subseteq X, \operatorname{cl}(A), \operatorname{int}(A)$ denote the closure of $A$, the interior of $A$ respectively. Let us recall the following definitions, which are useful in the sequel.

Definition 2.1. A subset $A$ of a topological space $(X, \tau)$ is called:

(1) $\alpha$-open if $A \subseteq \operatorname{int}(\operatorname{cl}(\operatorname{int}(A)))$ [9].

(2) semi open if $A \subseteq \operatorname{cl}(\operatorname{int}(A))$ [5].

(3) $\beta$-open if $A \subseteq \operatorname{cl}(\operatorname{int}(\operatorname{cl}(A)))[3]$.

(4) pre open if $A \subseteq \operatorname{int}(\operatorname{cl}(A))$ [7].

(5) $b$-open if $A \subseteq \operatorname{cl}(\operatorname{int}(A)) \cup \operatorname{int}(\operatorname{cl}(A))[1]$.

The following notion is crucial for our purposes

Received: 06.01.2016. In revised form: 09.05.2016. Accepted: 26.05.2016

2010 Mathematics Subject Classification. 54C05, 54C08, 54C10, 54 C60.

Key words and phrases. I- $\beta$-open sets, weakly I-open sets, I- $\alpha$-open sets, I-pre-open sets, I-semi open sets.

Corresponding author: Carlos Carpintero; carpintero.carlos@gmail.com 
Definition 2.2. An ideal $I$ on a topological space $(X, \tau)$ is a nonempty collection of subsets of $X$ which satisfies the following conditions:

(1) $A \in I$ and $B \subseteq A$ implies $B \in I$.

(2) $A \in I$ and $B \in I$ implies $A \cup B \in I$.

A topological space $(X, \tau)$ with an ideal $I$ on $X$, denoted by $(X, I, \tau)$, is called an ideal topological space.

Definition 2.3. A subset $A$ of an ideal topological space $(X, I, \tau)$ is called:

(1) semi open with respect to an ideal $I$ [8], denoted by $I$-semi open, if there exists $U \in \tau$ such that $U \backslash A \in I$ and $A \backslash \operatorname{cl}(U) \in I$.

(2) $\alpha$-open with respect to an ideal $I$ [11], denoted by $I$ - $\alpha$-open, if there exists $U \in \tau$ such that $U \backslash A \in I$ and $A \backslash \operatorname{int}(\operatorname{cl}(U)) \in I$.

(3) $\beta$-open with respect to an ideal $I$ [11], denoted by $I$ - $\beta$-open, if there exists $U \in \tau$ such that $U \backslash \operatorname{cl}(A) \in I$ and $A \backslash \operatorname{cl}(U) \in I$.

(4) pre-open with respect to an ideal $I$ [10], denoted by $I$-pre-open, if there exists $U \in \tau$ such that $A \backslash U \in I$ and $U \backslash \operatorname{cl}(A) \in I$.

In [11] and [10], the relationships between these types of sets are shown as follows:

Remark 2.1. For any ideal $I$, we have the following implications:

$I$ - $\alpha$-open $\Rightarrow I$-semi-open $\Rightarrow I$ - $\beta$-open $\Leftarrow I$-pre-open.

Also, this implications are not reversible and there are not exist relation between the $I-\alpha-$ open and $I$-pre-open.

The interest of these sets was to see if they could solve the following problem. Given $(X, \tau)$ and $A \subset X$ satisfies the property $\mathrm{W}$, if and only if, $\operatorname{cl}(A)$ satisfies it. It is well known that the property of compactness, connectedness are no applicable among others. It is easy to see that:

(1) If $c l(A)$ is $I$-Pre-open, then $A$ is $I$-Pre-open.

(2) If $c l(A)$ is $I$ - $\beta$-open, then $A$ is $I-\beta$-open.

(3) For the case of the sets $I$-semi-open, we can not said anything.

(4) For the case of the sets $I$ - $\alpha$-open, we can not said anything.

The following examples show that the converse of items (1) and (2) of the above implications are not necessarily true. Also we give examples for the items (3) and (4).

Example 2.1. Let $X=\{a, b, c\}$ with topology $\tau=\{\emptyset, X,\{b\},\{c\},\{b, c\}\}, I=\{\emptyset,\{a\}\}$. Then $\{b\}$ is $I$-pre-open, but $c l(\{b\})$ is not $I$-pre open, also $c l(\{b\})$ and $\{b\}$ are $I$-semi open sets.

Example 2.2. Let $X=\{a, b, c, d\}$ with topology $\tau=\{X, \emptyset$, $\{a, c\},\{a, b, c\}\}$ and $I=\{\emptyset\}$. Take $A=\{b, c\}$, then $C l(A)=X$ is I-semi open, but $A$ is not $I$-semi open. In the same form if we take $I=\{\emptyset,\{b\}\}$ and $A=\{b\}$, then $A$ is $I$-semi open but $\operatorname{cl}(A)$ is not.

Example 2.3. Let $X=\{a, b, c\}, \tau=\{X, \emptyset,\{c\}\}, I=\{\emptyset,\{b\}\}$ and $A=\{b\} . A$ is $I$ - $\alpha$-open, but $\operatorname{cl}(A)$ is not.

Example 2.4. Let $X=\{a, b, c, d\}, \tau=\{\emptyset, X,\{a\},\{b\},\{a, b\},\{a, c\}$, $\{a, b, c\}\}$ and $I=\{\emptyset,\{c\}\}$. If we take $A=\{c\}, A$ is $I$ - $\beta$-open but $c l(A)$ is not $I$ - $\beta$-open.

Finally, we show that in the case of the $I$ - $\alpha$-open, we can not said anything.

Example 2.5. Let $X=\{a, b, c, d\}, \tau=\{\emptyset, X,\{a\},\{b\},\{a, b\},\{a, b, c\}\}$ and $I=\{\emptyset\}$. If we take $A=\{a, b, d\}, \operatorname{cl}(A)$ is $I$ - $\alpha$-open but $A$ is not $I$ - $\alpha$-open. 
Example 2.6. Let $X=\{a, b, c, d\}, \tau=\{\emptyset, X,\{a\},\{b\},\{a, b\}\}$ and $I=\{\emptyset,\{c\}\}$. If we take $A=\{c\}, A$ is $I$ - $\alpha$-open but $c l(A)$ is not $I$ - $\alpha$-open.

In 2014, C. Carpintero et al. [12], showed that the Proposition 6 of [8], is not true in general and they gave a counterexample. In view of the above, it was thought to weaken the notion of $I$-semi-open set, in order to give a positive solution to the problem.

Definition 2.4. [12] Let $(X, I, \tau)$, A subset $A$ of $X$ is said to be weakly semi open with respect to an ideal $I$ (denoted by $I$-weakly semi open) if $A=\emptyset$ or if $A \neq \emptyset$ there exists an open set $U \neq \emptyset$ such that $U \backslash A \in I$.

It is easy to see that all semi open set are $I$-weakly semi open for any ideal $I$ and there are no guarantee that the elements of the ideal are $I$-weakly semi open.

Remark 2.2. For any ideal $I$, we have:

(1) If $A$ is any open set, then $A$ es $I$-weakly semi open.

(2) If $A$ is a semi open set, then $A$ is $I$-weakly semi open.

(3) If $A$ is an $I$-semi open set, then $A$ is not necessarily $I$-weakly semi open.

In [12] was proved the following theorem that give a positive answer to the problem, when working with $I$-weakly semi open.

Theorem 2.1. Let $(X, I, \tau)$ be an ideal topological space, $I \neq \emptyset$ an ideal, $\tau$ satisfies the finite intersection property and $A \subset X$ such that $C l(A) \neq X$, then $C l(A)$ is I-weakly semi open, if and only if, $A$ is I-weakly semi open.

In the same form, for the $I$ - $\beta$-open, we obtain a positive answer to the problem. As we can see.

Theorem 2.2. Let $(X, I \tau)$ be an ideal topological space and $A$ be a dense subset of $X . A$ is $I$ - $\beta$-open if and only if $\mathrm{cl}(A) I$ - $\beta$-open.

It is easy to see that the concept of $I$-weakly semi open is independent of all notions defined above, as is shown in the following examples.

Example 2.7. Let $X=\{a, b, c\}, \tau=\{\emptyset, X,\{a\},\{b, c\}\}$ and $I=\{\emptyset\}$. The set $A=\{a, b\}$ is $I$-weakly semi open, but is not $I$ - semi open.

Example 2.8. Let $X=\{a, b, c, d\}, \tau=\{\emptyset, X,\{a\},\{b\},\{a, b\},\{a, c\},\{a, b, c\}\}, I=\{\emptyset,\{b\}\}$ and $A=\{c\}$. Then $c l(A)=\{c, d\}$ is $I$-weakly semi open, but $c l(A)$ is not $I$ - $\beta$-open.

Example 2.9. Let $X=\{a, b, c, d\}, \tau=\{\emptyset, X,\{a, b\},\{c, d\}\}$ and $I=\{\emptyset\} . A=\{a\}$ is $I-\beta$ open, but $A$ is not $I$-weakly semi open.

Example 2.10. Let $X=\{a, b, c\}, \tau=\{\emptyset, X,\{a\},\{b\},\{a, b\}\}$ and $I=\{\emptyset,\{c\}\} . A=\{c\}$ is $I$ - $\beta$-open, but $c l(A)=\{c\}$ is not $I$-weakly semi open.

(1) If we take the set $A=\{a, c\}$ and $I=\{\emptyset,\{a\}\}$, then $A$ is $I$-weakly semi open but is not $I$-pre-open.

(2) If we take the set $A=\{b, c\}$ and $I=\{\emptyset\}$, then $A$ is $I$-weakly semi open but is not $I$ - $\alpha$-open.

In 2015, Arafa A. Nasef et al. [2], defined the notion of $I$ - $\beta$-open as follows

Definition 2.5. A subset $A$ of an ideal topological space $(X, I, \tau)$ is said to be $\beta$-open with respect to an ideal (written $I$ - $\beta$-open) if there exists a pre-open set $G$ such that $G \backslash A \in I$ and $A \backslash \operatorname{cl}(G) \in I$. 
Observe that now, we have two definitions of $I$ - $\beta$-open sets. From now on, we written $I$ - $\beta$-open $[\mathrm{R}]$ and $I$ - $\beta$-open $[\mathrm{A}]$, The first one is in the sense of Rodyna A. Hosny et al. and the second one in the sense of Arafa A. et al.

The principal result in [2], is the following theorem.

Theorem 2.3. Let $I$ be an ideal on a topological space $(X, \tau)$, where every non-empty pre-open subset of $X$ is dense and the collection of pre-open subsets of $X$ satisfies the finite intersection property. $A \subseteq X$ is $I$ - $\beta$-open [A] if and only if $\operatorname{cl}(A)$ is $I$ - $\beta$-open [A].

The following example shows the existence of such collections described in the above theorem.

Example 2.11. Let $X=\{a, b, c, d\}, \tau=\{\emptyset, X,\{a\},\{a, b\},\{a, b, c\}\}$ and $I$ any ideal. The collection of pre-open sets is $\{\emptyset, X,\{a\},\{a, b\},\{a, c\}$,

$\{a, d\},\{a, b, c\},\{a, b, d\},\{a, c, d\}\} . A$ is $I$ - $\beta$-open [A] if and only if $c l(A)$ is $I$ - $\beta$-open [A].

Our interest is to obtain the relations if there exists between the sets described above and the $I$ - $\beta$-open [A]

Theorem 2.4. Let $I$ be an ideal on a topological space $(X, \tau)$ and $A \subseteq X$.

(1) If $A$ is $I$ - $\alpha$-open then $A$ is $I$ - $\beta$-open [A].

(2) If $A$ is $I$-semi open then $A$ is $I$ - $\beta$-open [A].

(3) If $A$ is $I$-pre-open then $A$ is $I$ - $\beta$-open $[A]$.

The following examples show that the converse in the above theorem are not necessarily true.

Example 2.12. Let $X=\{a, b, c, d\}$ with topology $\tau=\{X, \emptyset,\{a, b, c\}$, $\{d\},\{a, b\},\{a, b, d\}\}$.

(1) If we take the set $A=\{b, c, d\}$ and $I=\{\emptyset\}$, then $A$ is $I$ - $\beta$-open [A] but is not I-pre-open.

(2) If we take the set $A=\{a\}$ and $I=\{\emptyset\}$, then $A$ is $I$ - $\beta$-open [A] but is not $I$-semi open.

(3) If we take the set $A=\{a\}$ and $I=\{\emptyset\}$, then $A$ is $I$ - $\beta$-open [A] but is not $I$ - $\alpha$-open.

The notions of $I$ - $\beta$-open [A] and $I$ - $\beta$-open [R] are independent, as we show in the following two examples.

Example 2.13. Let $X=\{a, b, c, d\}$ with topology $\tau=\{X, \emptyset,\{a, b, c\},\{d\},\{a, b\},\{a, b, d\}\}$. If we take the set $A=\{c, d\}$ and $I=\{\emptyset,\{c\}\}$, then $A$ is $I$ - $\beta$-open [R] but is not $I$ - $\beta$-open [A].

Example 2.14. Let $X=\{a, b, c\}$ with topology $\tau=\{X, \emptyset,\{a\},\{b, c\}\}$. If we take the set $A=\{a, c\}$ and $I=\{\emptyset\}$, then $A$ is $I$ - $\beta$-open [A] but is not $I$ - $\beta$-open [R].

The notions of $I$ - $\beta$-open [A] and $I$-weakly semi open sets are independent, as we show in the following two examples.

Example 2.15. Let $X=\{a, b, c, d\}$ with topology $\tau=\{X, \emptyset,\{a, b\}$,

$\{c\},\{a, b, c\},\{b\},\{b, c\}\}$. If we take the set $A=\{a, c, d\}$ and $I=\{\emptyset\}$, then $A$ is $I$-weakly semiopen but is not $I$ - $\beta$-open [A].

Example 2.16. Let $X=\{a, b, c, d\}$ with topology $\tau=\{X, \emptyset,\{a, b, c\}$,

$\{d\},\{a, b\},\{a, b, d\}\}$. If we take the set $A=\{b\}$ and $I=\{\emptyset\}$, then $A$ is $I$ - $\beta$-open [A] but is not $I$-weakly semi open. 
Finally, in the following diagram, we described all the relationship between the sets described above.

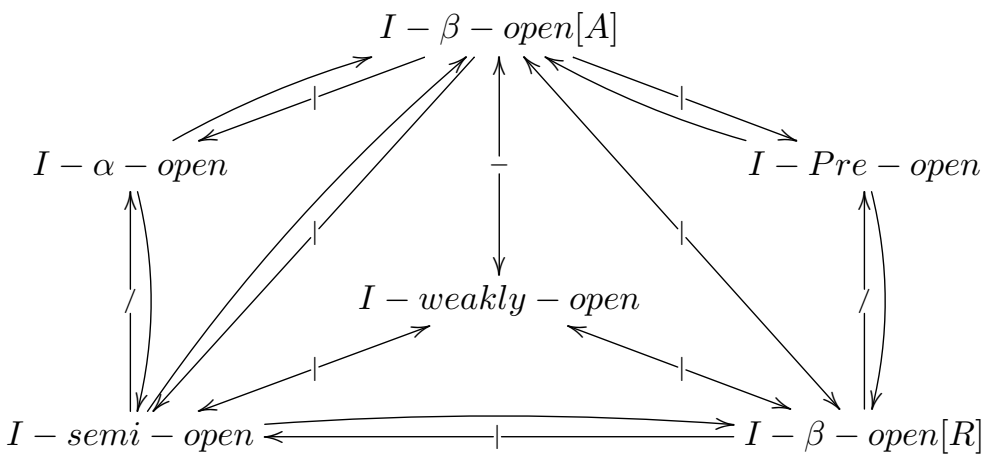

\section{I-b-OPEN SET}

We introduce the notion of $I-b$-open sets and study some of its characterizations and its relations with the sets defined before.

Definition 3.6. Let $(X, \tau, I)$ be an ideal topological space. $A \subset X$ is said to be an $I$-b-open set if there exist two open sets $U, V$ such that: $U \backslash A \in I, V \backslash \operatorname{cl}(A) \in I$ and $A \backslash(V \cup \operatorname{cl}(U) \in I$. Observe that if in the above definition, we take $I=\{\emptyset\}$, we recover the usual notion of $b$-open sets.

Theorem 3.5. Let $(X, \tau, I)$ be an ideal topological space. If $A \subseteq X$ is an $I$-b-open set and $I=\{\emptyset\}$, then $A$ is b-open set.

Theorem 3.6. Let $(X, \tau, I)$ be an ideal topological space and $A \subset X$. If $A$ is an $I$-b-open set, then $c l(A)$ is an $I$-b-open set.

Example 3.17. Let $X=\{a, b, c, d\}, \tau=\{X, \emptyset,\{a, c\},\{a, b, c\}\}$. Take $A=\{b, c\}$ and $I=\{\emptyset\}$. Observe that $\operatorname{cl}(A)=X$ is an $I$-b-open but $A$ is not.

Theorem 3.7. Let $(X, \tau, I)$ be an ideal topological space. If $A \subseteq X$ is an I-semi open set or an I-pre open set then $A$ is an I-b-open set.

The converse of the above theorem not necessarily is true. As we can see in the following example.

Example 3.18. Let $X=\Re$ the real line with the usual topology and $I=\{\emptyset\}$. Take $A=$ $[1,2] \cup((2,3) \cap Q)$. $A$ is $I$-b-open but neither $I$-semi open nor $I$-pre open.

The notions of $I$ - $\beta$-open $[R]$ and $I$-b-open are independent, as we can see in the following examples.

Example 3.19. In Example 2.9, the set $A=\{a\}$ is $I$ - $\beta$-open $[\mathrm{R}]$ but is not $I$ - $b$-open.

Example 3.20. In the Example 2.14, the set $A=\{a, c\}$ is $I$-b-open but is not $I$ - $\beta$-open [R].

The notions of $I$ - $\beta$-open [A] and $I$-b-open are independent, as we can see in the following examples.

Example 3.21. In Example 2.13, the set $A=\{c, d\}$ is $I$-b-open but is not $I$ - $\beta$-open [A].

Example 3.22. In Example 2.12, the set $A=\{b, c, d\}$ is $I$ - $\beta$-open [A] but is not $I$-b-open.

The notions of $I$-weakly semi open and $I$ - $b$-open are independent, as we can see in the following examples. 
Example 3.23. In Example 2.15, the set $A=\{a, c, d\}$ is $I$-weakly semi open but is not $I$-b-open.

Example 3.24. Let $X=\{a, b, c, d\}, \tau=\{\emptyset, X,\{a\},\{a, b\},\{a, b, c\}\}$ and $I=\{\emptyset,\{b\}$. The set $A=\{b\}$ is $I$-b-open but is not $I$-weakly semi open.

Finally we formulate the following open question: If $(X, \tau, I)$ is an ideal topological space, under what condition on the space and in the ideal we can said if $A \subset X$ such that $\operatorname{cl}(A)$ is an $I$ - $b$-open, then $A$ is an $I$ - $b$-open?

Acknowledgements. The authors thanks to the referees for their valuable comments and suggestions, specially in the notions of $I$ - $b$-open sets.

\title{
REFERENCES
}

[1] Andrijevic, D. b-open sets in topological spaces, Mathematica Vesnik, 48 (1996), 59-64

[2] Arafa, A. N., Mareay, R. and Michael, F. I., Idealization of Some Topological Concepts, Eur. J. Pure Appl. Math, 8 (2015), No. 3, 389-394

[3] Abd. El-Monsef, M. E., El-Deeb, S. N., and Mahmould, R. A., $\beta$-open sets and $\beta$-continuous mappings, Bull. Fac. Sci. Assiut. Univ, 12 (1983), 77-90

[4] Jafari, S. and Rajesh, N., Generalized closed sets with respect to and ideal, Eur. J. Pure Appl. Math, 4 (2011), No. 2, 147-151

[5] Levine, N., semi open sets and semi continuity in topological spaces, Amer. Math. Monthly, 70 (1963), 36-41

[6] Maki, H., Chandrasekhara Rao, K. and Nagoor Gani, A., On generalizing semi-open sets and preopen sets, Pure Appl. Math. Sci., 49 (1999), No. 1-2, 17-29

[7] Mashhour, A. S., Abd. El-Monsef, M. E. and El-Deeb, S. N., On precontinuous and weak precontinuous mappings, Proc. Math. Phys. Soc. Egypt, 53 (1982), 47-53

[8] Michael, F. I., On some open sets with respect to an ideal, Eur. J. Pure Appl. Math, 6 (2013), No. 1, 53-58

[9] Njastad, O., On some classes of nearly open sets, Pacific J. Math, 15 (1965), 961-970

[10] Rodyna, A. Hosny, Pre-open sets respect ideal, Eur. J. Scient. Research, 104 (2013), No. 1, 99-101

[11] Rodyna A. Hosny, and Deena, Al-Kadi, Types of Generalized Open Sets with Ideal, Int. J. Comput Appl., 80 (2013), No. 4, 97-112

[12] Rosas, E., Carpintero, C., Muñoz, A. and Pacheco, J., Some Remarks on Semi Open Sets with Respect to an Ideal, Eur. J. Pure Appl. Math, 7 (2014), No. 4, 437-441

\author{
${ }^{1}$ Departamento de Matemáticas \\ UNIVERSIDAD DE ORIENTE \\ CUMANÁ.VENEZUELA \\ Email address: carpintero.carlos@gmail.com \\ Email address: ennisrafael@gmail.com \\ ${ }^{2}$ UNIVERSIDAD DEL ATLÁNTICO \\ FaCUlTad de Ciencias BÁsicas \\ BARRANQUILla. COLOMBIA \\ Email address: jackelinepacheco25@gmail.com \\ Email address: ennisrafael@gmail.com
}

